\title{
ANTROPOMETHRIC PERFORMANCE CHARACTERISTICS OF FEMELE GREEK HANDBALL PLAYERS
}

\author{
Iordanis Giannakou, Sasho Chanev \\ National Sports Academy "Vassil Levski", Sofia, Bulgaria
}

\begin{abstract}
The objective of this study was to provide anthropometric performance characteristics of female Greek handball players. One hundred and one elite handball female players playing in 1st divisions were tested. Testing consisted of anthropometric measures of 25 indicators (height, hand range, upper limb length, lower limb length, length of the span, width of the span, shoulder width, chest circumference, respiratory difference, lap circumference arm, circumference of the forearm, circumference of the contra-arm, circumference of contracted arm, dynamometry of a strong hand, dynamometry of a week hand, weight, BMI, index, body mass\%, total body water \%, fat mass, muscle mass, metabolic age, bone mass and calories. Descriptive statistics, Correlation and Factor analysis, a multivariate correlation technique, were used to provide insight into the anthropometric profile of female Greek handball players. Logical and statistically significant strong correlations were found between height and hand range; length of upper limbs and hand range; between circumferences and body composition variables. Principal factor analysis identified fourth factors that explained 50, 20, 6 and 5\% of the variance in the dataset, respectively. These factors were interpreted as 1) "circumferences and body composition" factor, 2) "Body proportionality factor", 3) "Vital capacity" factor and 4) "Hand strength factor". The anthropometric characteristics can be used for optimization of the process of selection of young female handball players in Greece.
\end{abstract}

Key words: anthropometric profile, female handball players, selection

\section{INTRODUCTION}

Handball is an aerobic-anaerobic intermittent sport with high demands on the level of physical fitness. There are different playing positions on the court, based on a player's location on the field during either offensive (left wing, left back, centre back, right back, right wing, and pivot) or defensive (players are counted from the side to the centre of the field) phases. Each position has its own specificities.

Significant differences were found between player positions for some anthropometric characteristics (height and percentage body fat) but not for the physiological or performance characteristics.

It is found that the body composition of female handball players is an important prerequisite for their good performance, especially with regard to playing position-specific requirements (Vuleta, Milanović, \& Sertić, 1999; Vuleta, Milanović, et al., 2010; Urban \& Kandrač, 2011; Wagner, Finkenzeller, Würth, \& von Duvil-lard, 2014; Zapartidis, et al., 2009).
In the last two decades a number of research papers have been addressed to studying morphological characteristics of adult women handball players (Bon, et al., 2013; Čavala \& Katić, 2010; Granados, Izquierdo, Ibanez, Bonnabau, \& Gorostiaga, 2007).

Over the last few years, a new focus on the study of talent identification in team sports seems to have gained pre-eminence (Mohamed, et all., 2009, Elferink-Gemser, et all., 2007, Till, et all, 2011). The anthropometric, and physical performance characteristics are considered fundamental for talent identification and sport development and performance (Karapetrova, Soikov, Rankov, Soikov, 2010).

The present study tried to determine which anthropometric performance variables, by the factor analysis are the most significant for performance and can be used for selecting Greek female handball players.

The objective of this study is to provide the anthropometric performance characteristics of female 
Greek handball players. One hundred and one elite handball female players playing in 1st divisions were tested.

\section{METHODS}

\section{Participants}

One hundred and one elite handball female players playing in 1st divisions were tested. All the players took part in an anthropometric assessment.

\section{Procedures}

The players performed a battery of anthropometric tests at the end of the competitive season and as a part of their training programs. All assessments were carried out by handball expert and coaches and all the tests were completed on the same day.

Testing consisted of anthropometric measurement of 25 indicators - height, hand range, upper limb length, lower limb length, length of the span, width of the span, shoulder width, chest circumference, respiratory difference, lap circumference arm, circumference of the forearm, circumference of the contra-arm, circumference of contracted arm, strong hand grip strength, week hand grip strength measured with standard technics and weight, BMI ((BMI = body mass $/$ height $\left.2, \mathrm{~kg} \cdot \mathrm{m}-{ }^{2}\right)$, index (height - breast-weight circumference), body mass $\%$, total body water $\%$, fat mass, muscle mass, metabolic age, bone mass and calories measured with TANITA BODY COMPOSITION ANALYZER. SC-330.

\section{Statistical analysis}

Descriptive statistics, Correlation and Factor analysis, a multivariate correlation technique were performed to provide the anthropometric profile of female Greek handball players. The strength of correlation is set as follow: $\mathrm{r}=0$ - lack of correlations; $r$ till 0.3 - week; $r$ from 0.3 till 0.5 - moderate; $\mathrm{r}$ from 0.5 till 0.7 - significant; $\mathrm{r}$ from 0.7 till 0.9 - high, $r$ above 0.9 - very high and $r=1$ - functional. For studying factor structure and identifying main factors of the technical-tactical preparedness, the original correlation matrices was factorized by principle component analyse followed by Varimax orthogonal rotation method (Wainer/Braun, 1988).

The data were analysed following the standard procedures, using the statistical package for the Social Science (SPSS) v. 23 for MAC (IBM, New York, NY, USA). The level of error was set at $5 \%$, considering the differences with a $\mathrm{p} \leq 0.05$.

\section{RESULTS}

The anthropometric characteristics in the means, standard deviations and coefficients of variation of the female Greek handball players are presented in Table 1. Most of the variables are homogenous $(\mathrm{V} \leq 10-12 \%)(16)$ and the dispersion of the sample is low. The relatively homogenous variables are weight $(\mathrm{V}=15,3 \%)$ and $\mathrm{BMI}$ with $\mathrm{V}=14,5 \%$. The non-homogenous and with the high dispersion are the following variables: respiratory difference $(\mathrm{V}=37,7 \%)$, week hand grip strength $(\mathrm{V}=23,6 \%)$, index $(\mathrm{V}=55,2 \%)$, metabolic age $(\mathrm{V}=42,9 \%)$ and fat mass with the biggest coefficient $-\mathrm{V}=76,5 \%$. The figure 1 and 2 shown the dispersion of the variables divided in a two groups - morpho functional features characterizing body dimensions (Figure 1) and the features related to the body structure. (Figure 2).

The coefficients of correlations between the variables are shown in the Table $2(r \geq 0.25, \alpha \leq 0.1)$. There are 10 very high correlations, 4 of them are negative and 6 are positive. Some of them are logical as between BMI and Weight ( $r=.92)$, metabolic age and body mass $(\mathrm{r}=.90)$, calories and muscle mass $(\mathrm{r}=.94)$, calories and bonne mass $(\mathrm{r}=.94)$. More than 60 correlations are high. The variables, with more correlations are the following: weight, chest circumference, lap circumference arm, circumference of the forearm, circumference of the contra-arm, circumference of contracted arm, BMI, Index, body mass, total body water, muscle mass and calories. 
Table 1. Descriptive statistics of the variables $(n=101)$

\begin{tabular}{|l|c|c|c|c|c|c|}
\hline \multicolumn{1}{|c|}{ Variables } & $\begin{array}{c}\text { Measure } \\
\text { units }\end{array}$ & Min & Max & Mean & S & V\% \\
\hline 1. Height & $\mathrm{cm}$ & 153,0 & 177,5 & 167,3 & 5,1 & 3,0 \\
\hline 2. Hands range & $\mathrm{cm}$ & 149,0 & 181,0 & 168,1 & 6,6 & 4,0 \\
\hline 3. Upper limb length & $\mathrm{cm}$ & 62,5 & 81,0 & 72,8 & 3,3 & 4,5 \\
\hline 4. Lower limb length & $\mathrm{cm}$ & 81,0 & 107,0 & 96,7 & 4,3 & 4,5 \\
\hline 5. Length of the span & $\mathrm{cm}$ & 14,5 & 19,0 & 17,3 & 0,9 & 5,0 \\
\hline 6. Width of the span & $\mathrm{cm}$ & 19,0 & 24,0 & 21,4 & 1,1 & 5,0 \\
\hline 7. Shoulder width & $\mathrm{cm}$ & 35,5 & 44,0 & 38,9 & 1,8 & 4,6 \\
\hline 8. Chest circumference & $\mathrm{cm}$ & 66,5 & 96,0 & 76,8 & 6,1 & 8,0 \\
\hline 9. Respiratory difference & $\mathrm{cm}$ & 1,5 & 12,0 & 5,3 & 2,0 & 37,7 \\
\hline 10. Lap circumference arm & $\mathrm{cm}$ & 31,0 & 43,0 & 36,6 & 2,8 & 7,7 \\
\hline 11. Circumference of the forearm & $\mathrm{cm}$ & 22,0 & 36,5 & 26,8 & 2,8 & 10,4 \\
\hline 12. Circumference of the contra-arm & $\mathrm{cm}$ & 20,5 & 32,0 & 24,1 & 1,8 & 7,5 \\
\hline 13. Circumference of contracted arm & $\mathrm{cm}$ & 24,0 & 38,0 & 28,8 & 2,7 & 9,3 \\
\hline 14. Strong hand grip strength & $\mathrm{kg}$ & 10,1 & 30,6 & 19,7 & 4,5 & 22,8 \\
\hline 15. Week hand grip strength & $\mathrm{kg}$ & 8,4 & 30,0 & 17,3 & 4,1 & 23,6 \\
\hline 16. Weight & $\mathrm{kg}$ & 46,3 & 94,7 & 65,8 & 10,1 & 15,3 \\
\hline 17. BMI & $\mathrm{kg} / \mathrm{cm}{ }^{2}$ & 17,1 & 33,5 & 23,4 & 3,4 & 14,5 \\
\hline 18. Index & $\mathrm{index}$ & 23,2 & 52,9 & 24,7 & 15,3 & 55,2 \\
\hline 19. Body mass & $\%$ & 11,3 & 40,8 & 23,3 & 6,3 & 27,0 \\
\hline 20. Total body water & $\%$ & 44,7 & 67,7 & 57,3 & 4,7 & 8,2 \\
\hline 21. Fat mass & $\mathrm{index}$ & 1,0 & 7,0 & 1,7 & 1,3 & 76,5 \\
\hline 22. Muscle mass & $\%$ & 37,5 & 58,0 & 47,5 & 4,6 & 9,7 \\
\hline 23. Metabolic age & $\mathrm{age}$ & 12,0 & 41,0 & 17,7 & 7,6 & 42,9 \\
\hline 24. Bone mass & $\mathrm{kg}$ & 2,0 & 3,0 & 2,5 & 0,2 & 8,0 \\
\hline 25. Calories & $\mathrm{cal}$ & 2267,0 & 3341,0 & 2798,1 & 255,3 & 9,1 \\
\hline
\end{tabular}

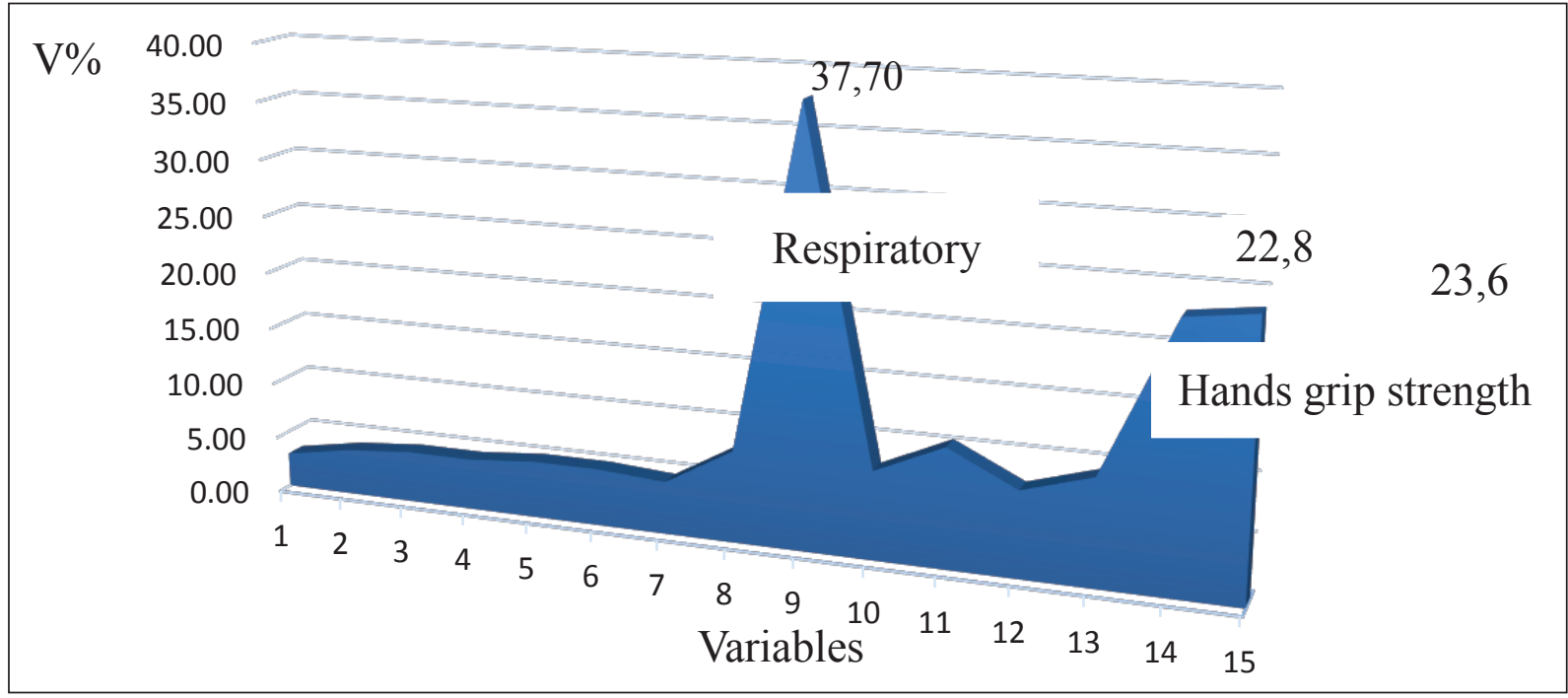

Figure 1. Dispersion of the morphofunctional features characterizing body dimensionsw 


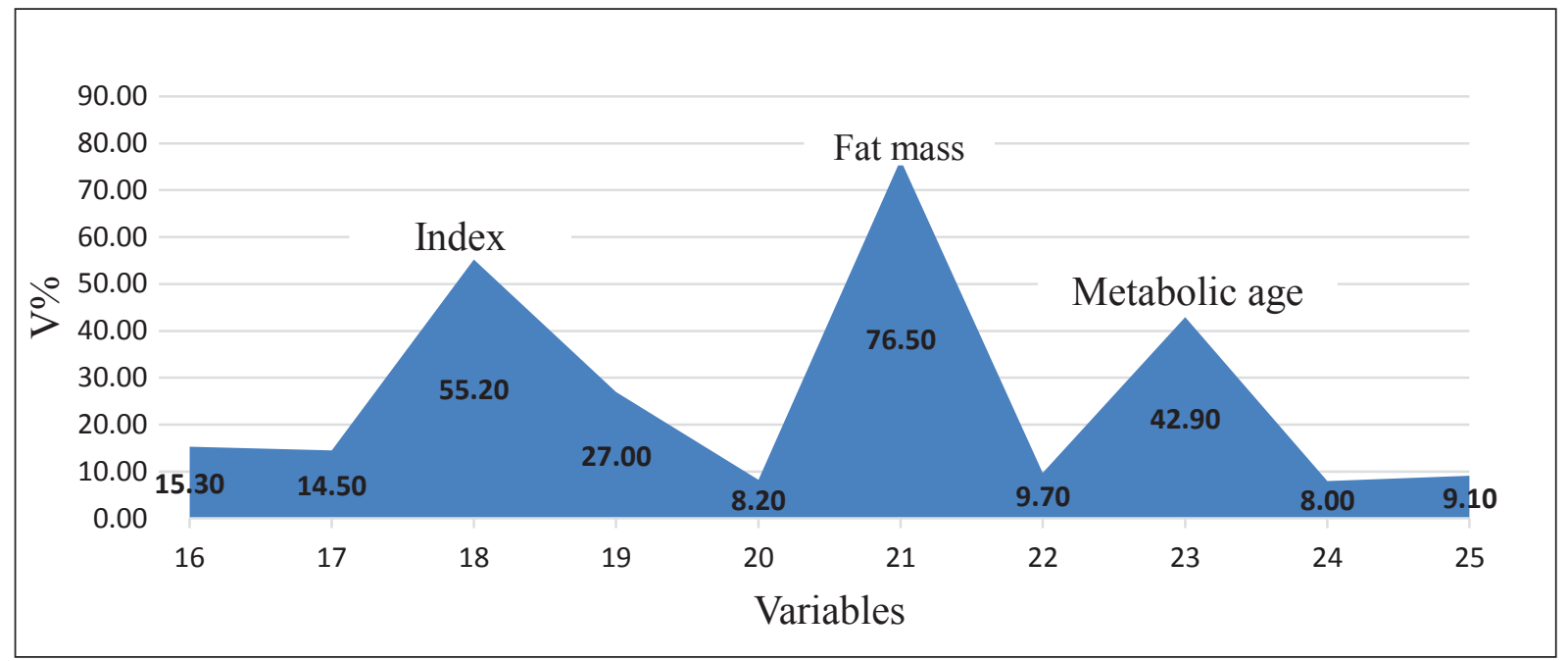

Figure 2. Dispersion of the variables related to the body structure

In Table 3, the factor structure of anthropometric profile of female Creek handball players is presented. From column I to IV, the factor loading of the extracted factors are presented. The last two columns give an idea of the community of each indicator and the initial not explained variance by the extracted factors.

In the last row of the table, under each factor is indicated the percentage of the total variance explained by it, which makes possible to evaluate and compare the individual factors.

The analysis of Figure 3 shows that the extracted factors explain a rather high percentage of the variance of the studied phenomenon - anthropometric profile of female Geek handball players. The relative proportions of the explained initial variance of each factor are as follows: for the first $-38.2 \%$, for the second $-18.3 \%$, for the third $-17.6 \%$ and for the fourth $-7.62 \%$.

\section{DISCUSSION}

According to Ziv, Lidor, (2009) the anthropometric characteristics can be used as a selection criterion for selection of players among the youth handball players. Handball involves frequent body contact and several high-intensity actions as part of a match play (Póvoas et al., 2012). Knowledge of the physical characteristics of handball players can provide insight into the individual factors which influence the players' performance in the game (Hasan et al., 2007).

In the present study, the Greek female handball players are highly homogenous concerning body structure parameters and non-homogenous for some morpho functional characteristics of the body dimensions. In the talent selection, the anthropometry and physical condition is considered essential factors (Hoare, Warr, 2000; Keogh, Weber, Dalton, 2003; Reilly, Bangsbo, Franks, 2000). However, these could be affected by the maturity state and not take into account the other important performance factors, such as the cognitive ability or emotional competence (Burgess, Naughton, 2010). Biological maturation on the selection process is complex and has several risks. Handball coaches should be aware of the maturity-selection that is based on the studied criteria that is not recommended as a long-term development of performance (Matthys, Fransen, Vaeyens, Lenoir, Philippaerts, 2013).

The female Greek handball players are taller bet-

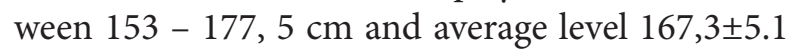
$\mathrm{cm}$; weight on average $65,8 \pm 10,1 \mathrm{~kg}$, normal BMI (Mean $=23.4 \pm 3,4 \mathrm{~kg} / \mathrm{cm}^{2}$ ), and have a low level of muscle mass (Mean $=47,5 \pm 4,6 \%$ ), but higher level of total body water (Mean $=57,3 \pm 4,7 \%$ ).

Certain anthropological characteristics have significant influence on the position-related performance in sports (Sibila and Pori, 2009). It would appear that the anthropometric characteristics of elite handball players appear to be different across positions. Williams \& Reilly (2000) stated, that the anthropometric characteristics such as body stature, body mass, limb girths and bone circumferences provide as interesting information, but they are related to performance in a complicated way. 


\begin{tabular}{|c|c|c|c|c|c|c|c|c|c|c|c|c|c|c|c|c|c|c|c|c|c|c|c|c|}
\hline 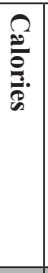 & 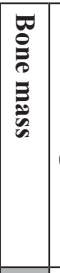 & 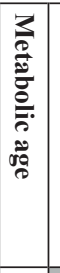 & 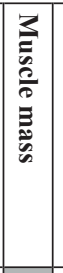 & 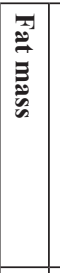 & 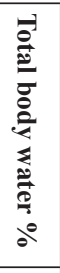 & 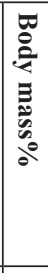 & $\bar{E}$ & 噪 & & 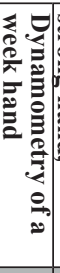 & $\mid$ & 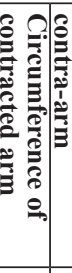 & 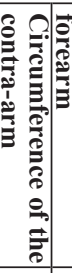 & 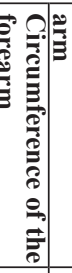 & 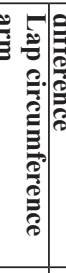 & $\begin{array}{l}2 \\
0 \\
0\end{array}$ & 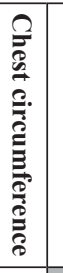 & 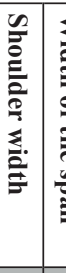 & 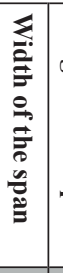 & 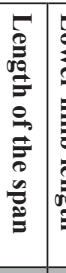 & 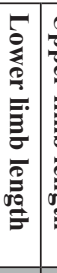 & 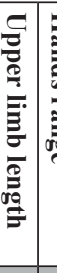 & 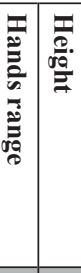 & Variables \\
\hline $\begin{array}{l}0 \\
+\infty \\
\infty\end{array}$ & 㸝 & $\begin{array}{l}0 \\
\infty\end{array}$ & î & 品 & $\stackrel{\dot{b}}{\dot{\omega}}$ & 号 & $\because$ & $\mid$\begin{tabular}{l}
$\dot{1}$ \\
\hdashline \\
$\dot{1}$
\end{tabular} & $\stackrel{0}{u}_{u}$ & $\stackrel{\sim}{\omega}_{\omega}^{0}$ & 怘 & $\stackrel{\circ}{10}$ & 옹 & $\stackrel{\circ}{\circ}$ & $\stackrel{0}{\sqcup}$ & $\begin{array}{l}0 \\
\infty\end{array}$ & $\stackrel{\circ}{\omega}$ & $\begin{array}{l}0 \\
\text { : } \\
\text { N }\end{array}$ & $\begin{array}{ll}0 \\
\text { 点 }\end{array}$ & 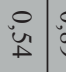 & 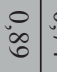 & $\stackrel{\vec{A}}{\vec{A}}$ & \begin{tabular}{c|c}
0 & - \\
$\infty$ & $\infty$
\end{tabular} & Height \\
\hline $\begin{array}{l}0 \\
\text { 品 }\end{array}$ & $\mid \begin{array}{l}0 \\
\dot{\omega} \\
\dot{\omega}\end{array}$ & 唄 & 品 & $\begin{array}{l}0 \\
\text { ur } \\
0\end{array}$ & $\stackrel{0}{0}$ & $\stackrel{0}{8}$ & $\begin{array}{l}1 \\
0 \\
0 \\
0\end{array}$ & $\begin{array}{l}\circ \\
\stackrel{1}{\perp}\end{array}$ & 品 & 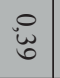 & $\begin{array}{l}0 \\
\text { uे }\end{array}$ & $\stackrel{0}{\infty}$ & $\stackrel{0}{\infty}$ & $\stackrel{0}{0}$ & 怘 & $\stackrel{\circ}{\square}$ & $\begin{array}{l}\stackrel{0}{0} \\
\stackrel{\sim}{0}\end{array}$ & $\begin{array}{l}0 \\
\text { a } \\
\text { a }\end{array}$ & 选 & : & $\stackrel{0}{0}$ & $\begin{array}{ll}0 \\
\infty \\
\infty \\
\infty\end{array}$ & 5 & Hands range \\
\hline 足 & $\mid \begin{array}{l}0 \\
\dot{1} \\
\infty\end{array}$ & $\stackrel{0}{\omega}$ & $\begin{array}{l}0 \\
\pm \\
\pm\end{array}$ & ○ & 主 & $\stackrel{0}{\not}$ & $\begin{array}{l}1 \\
0 \\
8\end{array}$ & $\stackrel{0}{\circ}$ & $\stackrel{\circ}{.}$ & $\stackrel{\circ}{\circ}$ & $\begin{array}{l}0 \\
\stackrel{1}{a} \\
\end{array}$ & $\stackrel{0}{N}$ & $\underset{\sim}{0}$ & 苂 & $\underset{N}{\stackrel{D}{\perp}}$ & $\stackrel{0}{u}$ & $\stackrel{\circ}{\Xi}$ & ن & 总 & $\stackrel{\circ}{\circ}$ & $\stackrel{\circ}{\sim}$ & $\dot{8}$ & & Upper limb length \\
\hline 怘 & $\begin{array}{l}0 \\
\pm \\
\pm\end{array}$ & $\begin{array}{l}0 \\
0 \\
0\end{array}$ & $\begin{array}{l}0 \\
\text { o } \\
\text { to }\end{array}$ & $\begin{array}{l}0 \\
0 \\
0\end{array}$ & $\stackrel{b}{\omega}$ & $\because 0$ & $\stackrel{0}{\square}$ & $\stackrel{0}{=}$ & 見 & 옥 & ్ & $\begin{array}{l}\circ \\
\text { \& }\end{array}$ & $\stackrel{0}{\circ}$ & $\begin{array}{l}0 \\
\text { us }\end{array}$ & $\begin{array}{l}0 \\
0 \\
0\end{array}$ & $\begin{array}{l}0 \\
0 \\
0\end{array}$ & $\begin{array}{l}0 \\
\stackrel{0}{0} \\
\stackrel{0}{0}\end{array}$ & 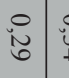 & $\underset{\stackrel{\leftrightarrow}{\perp}}{\circ}$ & 嗢 & 5 & & & Lower limb length \\
\hline 只 & 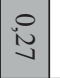 & $\begin{array}{l}\circ \\
0\end{array}$ & $\begin{array}{l}0 \\
\mathbb{N} \\
\infty\end{array}$ & $\ddot{8}$ & $\begin{array}{l}1 \\
\stackrel{\infty}{\infty}\end{array}$ & 足 & $\begin{array}{l}0 \\
8\end{array}$ & $\mid \begin{array}{l}0 \\
0 \\
0 \\
0\end{array}$ & $\stackrel{0}{0}$ & $\stackrel{\circ}{\tilde{\omega}}$ & 足 & $\stackrel{0}{0}$ & $\underset{\sim}{\stackrel{\sim}{\perp}}$ & $\begin{array}{l}\circ \\
\stackrel{0}{9}\end{array}$ & $\stackrel{0}{\omega}$ & $\begin{array}{l}\circ \\
\stackrel{0}{9}\end{array}$ & $\begin{array}{l}0 \\
\stackrel{0}{0}\end{array}$ & 唨 & $\begin{array}{l}0 \\
\text { 足 }\end{array}$ & 5 & & & & Length of the span \\
\hline$\dot{\omega}_{\infty}^{0}$ & $\mid$\begin{tabular}{l|}
0 \\
0 \\
$\dot{\omega}$ \\
\end{tabular} & $\begin{array}{l}0 \\
\dot{0} \\
\text { u }\end{array}$ & $\begin{array}{l}0 \\
0 \\
0 \\
0\end{array}$ & $\begin{array}{l}\dot{1} \\
\text { Oo } \\
0\end{array}$ & $\begin{array}{l}\dot{1} \\
\stackrel{\infty}{0}\end{array}$ & 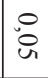 & $\begin{array}{l}\dot{0} \\
\dot{N}\end{array}$ & 品 & i & $\stackrel{\circ}{\stackrel{\sim}{y}}$ & 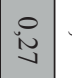 & 芫 & 芫 & $\stackrel{0}{\circ}$ & $\stackrel{\circ}{\square}$ & i & $\begin{array}{l}\stackrel{0}{\tilde{O}} \\
\end{array}$ & $\stackrel{\circ}{+}$ & 5 & & & & & Width of the span \\
\hline : & : & 怘 & $\therefore$ & $\stackrel{0}{0}$ & $\begin{array}{l}\dot{0} \\
\text { NU }\end{array}$ & $\underset{\sim}{\sim}$ & $\begin{array}{l}1 \\
0 \\
+ \\
+\end{array}$ & 足 & 品 & 只 & 怘 & 怘 & $\stackrel{\text { ¿ }}{\circ}$ & $\underset{\infty}{\infty}$ & $\stackrel{\circ}{+}$ & $\stackrel{1}{0}$ & 亚 & 5 & & & & & & Shoulder width \\
\hline$\stackrel{\circ}{a}$ & $\stackrel{0}{\beth}$ & $\begin{array}{l}0 \\
y \\
y\end{array}$ & $\because$ & $\frac{0}{N}$ & $\begin{array}{l}\dot{0} \\
\dot{a}\end{array}$ & $\stackrel{0}{0}$ & $\begin{array}{l}0 \\
0 \\
0 \\
\text { un }\end{array}$ & : & $\stackrel{0}{\infty}$ & $\stackrel{0}{\omega}$ & $\underset{\sim}{\stackrel{\sim}{\omega}}$ & $\stackrel{0}{\infty}$ & $\stackrel{\circ}{\sim}$ & $\stackrel{0}{\infty}$ & $\stackrel{0}{0}$ & 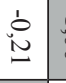 & 5 & & & & & & & $\begin{array}{l}\text { Chest } \\
\text { circumference }\end{array}$ \\
\hline$\stackrel{\dot{0}}{\infty}$ & $\begin{array}{l}0 \\
. \\
\omega\end{array}$ & $\stackrel{0}{=}$ & $\begin{array}{l}\dot{0} \\
0 \\
\dot{u}\end{array}$ & $\begin{array}{l}0 \\
0 \\
0\end{array}$ & $\begin{array}{l}0 \\
\text { u }\end{array}$ & 家 & 0 & $\begin{array}{l}0 \\
0 \\
0\end{array}$ & $\stackrel{\dot{\omega}}{\omega}$ & $\stackrel{0}{N}$ & 芯 & $\stackrel{\dot{n}}{\circ}$ & 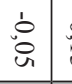 & $\stackrel{0}{\omega}$ & $\stackrel{0}{0}$ & 5 & & & & & & & & $\begin{array}{l}\text { Respiratory } \\
\text { difference }\end{array}$ \\
\hline$\stackrel{\infty}{\infty}$ & $\ddot{0}$ & ப் & $\stackrel{0}{u}$ & ¿ू & ì & $\stackrel{0}{u}$ & $\begin{array}{l}1 \\
\infty \\
\infty \\
\infty\end{array}$ & $\mid \begin{array}{l}0 \\
\infty \\
-1\end{array}$ & $\begin{array}{l}\infty \\
\infty \\
\infty\end{array}$ & $i_{\dot{w}}^{0}$ & $\stackrel{0}{\omega}$ & $\stackrel{0}{0}$ & 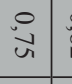 & 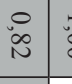 & 5 & & & & & & & & & $\begin{array}{l}\text { Lap circumference } \\
\text { arm }\end{array}$ \\
\hline$\stackrel{0}{\rightarrow+}$ & $\dot{\infty}$ & $\dot{n}$ & $\because$ & $\ddot{0}$ & $\stackrel{\dot{y}}{y}$ & $\ddot{\theta}_{0}^{0}$ & $\begin{array}{l}1 \\
0 \\
\infty \\
0\end{array} \mid$ & $\begin{array}{l}0 \\
\infty \\
\infty\end{array}$ & $\begin{array}{l}\infty \\
\infty\end{array}$ & 岕 & $\stackrel{\circ}{\omega}$ & $\stackrel{0}{\circ}$ & $\stackrel{\infty}{\infty}$ & 5 & & & & & & & & & & $\begin{array}{l}\text { Circumference of } \\
\text { the forearm }\end{array}$ \\
\hline$\stackrel{9}{9}$ & : & $\stackrel{\circ}{0}$ & $\because 8$ & $\stackrel{0}{0}$ & $\stackrel{b}{y}$ & $\stackrel{0}{w}$ & $\dot{b}$ & $\stackrel{0}{0}$ & $\begin{array}{l}0 \\
\infty \\
\infty\end{array}$ & $\begin{array}{l}0 \\
\stackrel{1}{*}\end{array}$ & 足 & $\begin{array}{l}0 \\
\infty \\
+\infty\end{array}$ & $\ddot{8}$ & & & & & & & & & & & $\begin{array}{l}\text { Circumference of } \\
\text { the contra-arm, }\end{array}$ \\
\hline$\stackrel{0}{w}$ & $\therefore$ & $\stackrel{\circ}{\sim}$ & 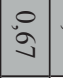 & $\ddot{0}$ & $\stackrel{\dot{a}}{\pi}$ & $\stackrel{\text { บy }}{0}$ & $\begin{array}{l}0 \\
\infty \\
\infty \\
+\end{array}$ & $\begin{array}{l}0 \\
\infty \\
\infty\end{array}$ & $\begin{array}{l}0 \\
\infty \\
\infty\end{array}$ & 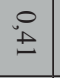 & $\stackrel{0}{\sim}$ & 5 & & & & & & & & & & & & $\begin{array}{l}\text { Circumference of } \\
\text { contracted arm }\end{array}$ \\
\hline$\underbrace{\sim}_{\sim}$ & $\mid \begin{array}{l}0 \\
0 \\
\text { in }\end{array}$ & 足 & $\begin{array}{l}0 \\
0 \\
0 \\
0\end{array}$ & $\stackrel{\circ}{\text { N }}$ & $\stackrel{i}{\stackrel{1}{N}}$ & 怘 & $\begin{array}{l}1 \\
0 \\
\\
\end{array}$ & i & $\stackrel{\infty}{\infty}_{\infty}$ & $\stackrel{\circ}{a}$ & 5 & & & & & & & & & & & & & $\begin{array}{l}\text { Dynamometry of a } \\
\text { strong hand }\end{array}$ \\
\hline$\stackrel{0}{\omega}^{\dot{\omega}}$ & 足 & $\stackrel{0}{\omega}$ & $\stackrel{0}{\omega}_{0}$ & i & $\underset{\infty}{\infty}$ & i & $\begin{array}{l}1 \\
0 \\
i \\
0\end{array}$ & $\stackrel{0}{0}$ & $\stackrel{\circ}{\doteq}$ & 5 & & & & & & & & & & & & & & $\begin{array}{l}\text { Dynamometry of a } \\
\text { week hand }\end{array}$ \\
\hline 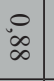 & $\begin{array}{l}0 \\
\infty \\
\infty\end{array}$ & $\begin{array}{l}0 \\
\infty\end{array}$ & $\begin{array}{l}0 \\
\infty \\
\infty\end{array}$ & $\stackrel{0}{u}$ & $\underset{\infty}{\infty}$ & $\infty$ & $\begin{array}{l}1 \\
0 \\
0 \\
0\end{array}$ & 音 & 5 & & & & & & & & & & & & & & & Weight \\
\hline$\stackrel{\circ}{\omega}$ & $\dot{\text { uे }}$ & $\begin{array}{l}\infty \\
\infty \\
\infty\end{array}$ & $\therefore$ & $\stackrel{0}{\infty}$ & $\underset{\infty}{\infty}$ & $\infty$ & $\begin{array}{l}1 \\
0 \\
0 \\
\infty\end{array}$ & 5 & & & & & & & & & & & & & & & & BMI \\
\hline$\stackrel{\dot{c}}{w}$ & $\begin{array}{l}\dot{0} \\
\dot{\omega}\end{array}$ & $\begin{array}{l}1 \\
0 \\
\infty \\
N\end{array}$ & $\begin{array}{l}\dot{0} \\
\vdots \\
\text { uे }\end{array}$ & $\dot{y}$ & $\stackrel{\infty}{\infty}$ & $\begin{array}{l}0 \\
0 \\
0 \\
1\end{array}$ & $\dot{8}$ & & & & & & & & & & & & & & & & & Index \\
\hline 总 & 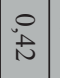 & $\dot{8}$ & $\begin{array}{l}0 \\
\pm\end{array}$ & $\begin{array}{l}0 \\
\infty \\
\infty\end{array}$ & io & 5 & & & & & & & & & & & & & & & & & & Body mass\% \\
\hline $\begin{array}{l}\dot{0} \\
\text { in } \\
\text { }\end{array}$ & $\begin{array}{l}1 \\
0 \\
\dot{N} \\
\end{array}$ & $\begin{array}{l}1 \\
\infty \\
\infty \\
\infty\end{array}$ & $\begin{array}{l}1 \\
0 \\
+1 \\
0\end{array}$ & $\dot{y}$ & 5 & & & & & & & & & & & & & & & & & & & Total body water \% \\
\hline$\stackrel{\circ}{ \pm}$ & $\stackrel{0}{.}$ & : & $\underset{\sim}{0}$ & 5 & & & & & & & & & & & & & & & & & & & & Fat mass \\
\hline$\dot{0}$ & $\begin{array}{l}0 \\
\dot{0} \\
\infty\end{array}$ & 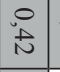 & 5 & & & & & & & & & & & & & & & & & & & & & Muscle mass \\
\hline in & $\begin{array}{l}0 \\
0 \\
\dot{\omega} \\
\end{array}$ & 5 & & & & & & & & & & & & & & & & & & & & & & Metabolic age \\
\hline i̊ & 8 & & & & & & & & & & & & & & & & & & & & & & & Bone mass \\
\hline & & & & & & & & & & & & & & & & & & & & & & & & Calories \\
\hline
\end{tabular}


Moreover, Reilly, Bangsbo, Franks, (2000) write that the size of that correlation can be modified through different levels of maturation, as anthropometric characteristics are affected by heredity in different ways. Our results sometimes showed no logical correlations and unstable factor structure of the anthropometric profile of the tested Greek female handball players. So, some anthropometric characteristics, such as body dimensions and body structure can be unreliable predictors of a future performance potential because of their high variability during puberty (Pearson, Naughton, Torode, 2006).

The present study contains some limitations. It shows data for the general players from the championship who had already been selected.

\section{CONCLUSION}

The present study examined the effectiveness of a battery of anthropometric tests for determining the anthropometric performance profile of Greek female handball players. The anthropometric characteristics should be included in any selection testing of young handball players. However, the selection should not be restricted to anthropometry, especially in young ages where maturation is involved. However, the measurement of motor physical characteristics, in combination with specific handball game tests should also be included in a selection procedure.

\section{REFFERENCES}

Bon, M., Pori, P., \& Šibila, M. (2013). Specialised handball classes in Slovenia: differences in identified characteristics between men and women junior participants. In EHF (Ed.), Women and handball. Scientific and practical approaches 2nd EHF Scientific Conference, pp. 324-328.

Čavala \& Katić. (2010). Morphological, Motor and Situation-Motor Characteristics of Elite Female Handball Players According to Playing Performance and Position. Coll. Antropol. 34, 4: 1355-1361

Elferink-Gemser, MT et all. (2007). Multidimensional performance characteristics and standard of performance in talented youth field hockey players: a longitudinal study. J Sports Sci.;25(4):481-9.

Granados, Izquierdo, Ibanez, Bonnabau, \& Gorostiaga. (2007). Differences in Physical Fitness and Throwing Velocity Among Elite and Amateur Female Handball Players. Int J Sports Med; 28(10): 860-867.
Hasan AAA, Rahaman JA, Cable NT, Reilly T. (2007). Anthropometric profile of elite male handball players in Asia. Biol Sport, 24(1): 3-12.

Haugsdorf: Hofer.Burgess, D.J.; Naughton, G.A. (2010). Talent development in adolescent team sports: A review. Int. J. Sports. Physiol. Perform, 5, 103-116. (PubMed)

Hoare, D.G.; Warr, C.R. (2000). Talent identification and women's soccer: An Australian experience. J. Sports Sci., 18, pp. 751-758. [CrossRef] [PubMed].

Karapetrova, R., G.Stoikov, Kr. Rankov, St. Stoikov (2010).Anthropometric parameters of the best javelin throwers - men and women. Actual trends of physical culture. Sport and Science, exp. Number, Sofia, pp. 119124.

Keogh, J.W.; Weber, C.L.; Dalton, C.T. (2003). Evaluation of anthropometric, physiological, and skill-related tests for talent identification in female field hockey. Can. J. Appl. Physiol. 28, pp. 397-409. [CrossRef] [PubMed]

Matthys, S.P.; Fransen, J.; Vaeyens, R.; Lenoir, M.; Philippaerts, R. (2013). Differences in biological maturation, anthropometry and physical performance between playing positions in youth team handball. J. Sports Sci. 31, 1344-1352. [CrossRef] [PubMed]

Mohamed H, et all. (2009). Anthropometric and performance measures for the development of a talent detection and identification model in youth handball. Journal of Sports Sciences. Volume 27, Issue 3.

Pearson DT, Naughton GA, Torode M. (2006). Predictability of physiological testing and the role of maturation in talent identification for adolescent team sports. J Sci Med Sports; 9: 277-87

Póvoas SC, Seabra AF, Ascensão AA, Magalhães J, Soares JM, Rebelo AN. (2012). Physical and physiological demands of elite team handball. J Strength Cond Res. 26(12):3365-3375.

Reilly, T.; Bangsbo, J.; Franks, A. (2000). Anthropometric and physiological predispositions for elite soccer. J. Sports Sci., 18, 669-683. [CrossRef] [PubMed].

Sibila M, Pori P. (2009). Position-related differences in selected morphological body characteristics of top-level handball players. Coll Antropol, 33(4): 1079-1086

Till K, C et all. (2011). Using anthropometric and performance characteristics to predict selection in junior UK Rugby League players. Journal of Science and Medicine in Sport, Volume 14, Issue 3, Pages 264-269.

Urban \& Kandrač. (2011). Position-Related Change in Somatotypes of Top Level Male Handball Players: 1980 and 2010. In EHF Scientific Conference: Science and Expertise in Handball (Scientific and practical approaches). pp. 214-218.

Vuleta, Milanović, \& Sertić. (1999). Latent structure of 


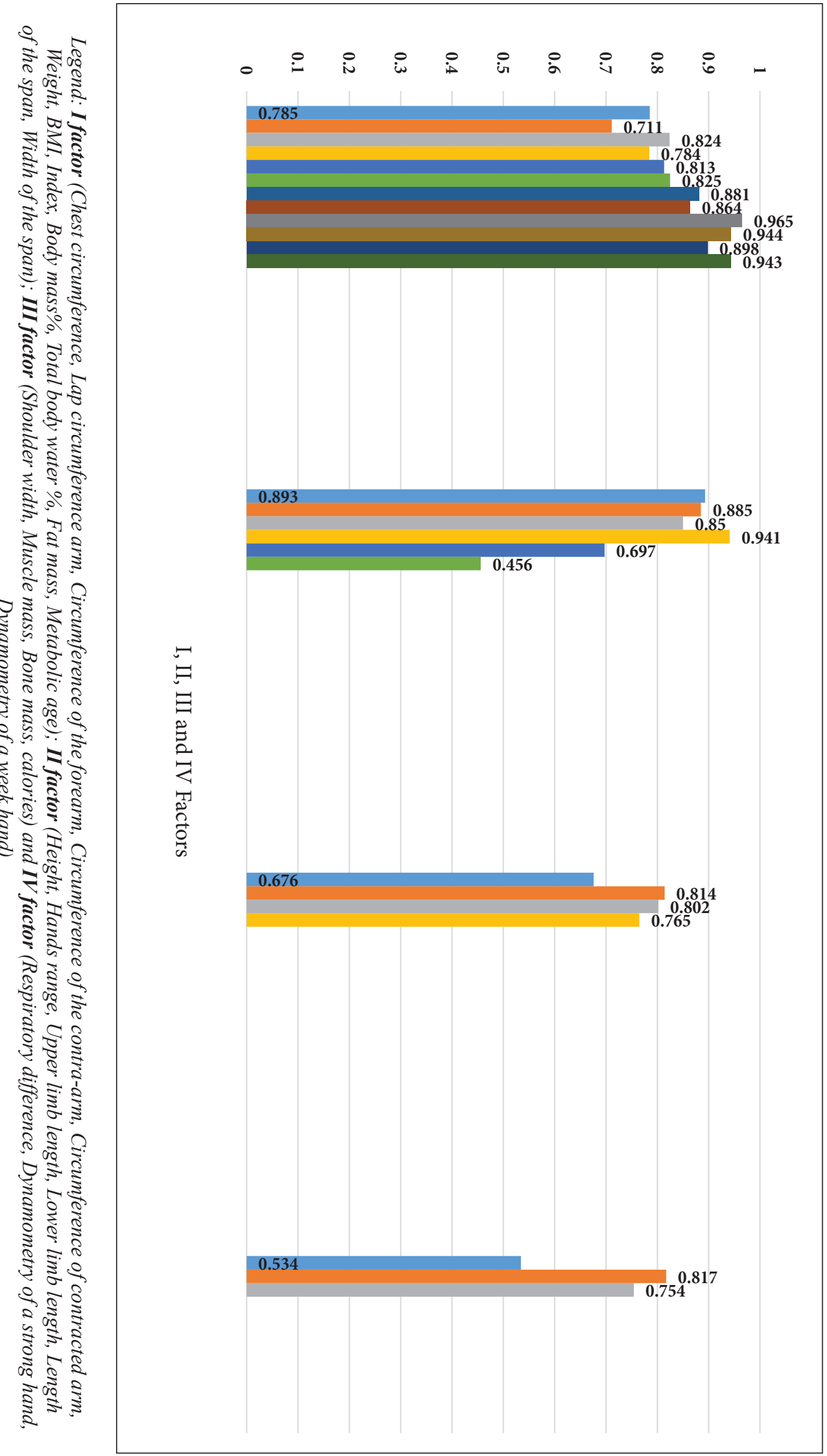

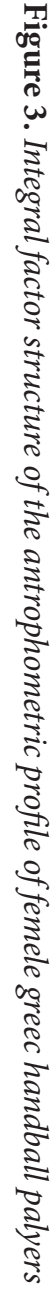


spatial, phasic, positional and movement characteristic Williams AM, Reilly T. (2000). Talent identification and of the handball game. Kinesiology: international journal development in soccer. J Sports Sci 18: 657-67.

of fundamental and applied kinesiology (1331-1441) 31, Zapartidis, et al. (2009). Profile of Young Handball pp. 37-53

Players by Playing Position and Determinants of Ball

Vuleta, Milanović, et al. (2010). Fitness Profiling in Throwing Velocity. Journal of Human Kinetics. Volume

Handball: Physical and Physiological Characteristics of 27, pp. 17-30.

Elite Players. Collegium antropologicum, Vol. 34 No. 3. Ziv, G.; Lidor, R. (2009). Physical attributes, physio-

Wagner, Finkenzeller, Würth, \& von Duvil-lard. (2014). logical characteristics, on-court performances and nutri-

Individual and Team Performance in Team-Handball: $A$ tional strategies of female and male basketball players.

Review. J Sports Sci Med.; 13(4) pp. 808-816.

Sports Med., 39, pp. 547-568. [CrossRef] [PubMed] 